\title{
Retrospective analysis on adverse drug reactions prevalence in the female population of Tertiary Care Hospital
}

\author{
Rangeel Singh Raina ${ }^{1}$, Sona Bharti Kaushal,** \\ ${ }^{1}$ Professor, ${ }^{2}$ Associate Professor, Dept. of Pharmacology, Government Doon Medical College, Dehradun, Uttarakhand, India
}

*Corresponding Author:

Email: sonakaushal11@gmail.com

\begin{abstract}
Introduction: Adverse drug reactions seems to be a major problem in women than men.

Objective: This study as planned to assess the incidence rate, clinical manifestations, and causative agents for adverse drug reactions (ADRs) in female patients.

Materials and Methods: This study was a retrospective study in which analysis of the clinical variables related to all suspected cases of ADRs reported in female patients of the tertiary centre hospital.

Results: Out of 206 patients reported with ADR in between period of 2012 to 2015, $110(53.4 \%)$ were female whereas only $46.6 \%$ were male patients. Although Mean age of female patients affected from ADRs was 31.53 years and most of ADRs were reported in the age group of 20-39 years (41.8\%).Department of dermatology has reported maximum number of ADRs in females $(51,46.36 \%)$, followed by medicine $(21,23.6 \%)$ and Surgery $(19,17.02 \%)$. Amongst 85 ADRs cases reported due to antimicrobial, betalactams $(31,36.5 \%)$ and quinolones $(17,20 \%)$ were the mostly responsible, followed by aminoglycosides (11, $12.9 \%)$. Skin was affected more frequently (69.6\%), followed by gastrointestinal (14.9\%).

Conclusion: Adverse drug reactions in female seems to be a significant problem and we need to evaluates the physiological and other cofactors to understand the high occurrence of adverse drug reaction in females.
\end{abstract}

Keywords: Adverse Drug Reactions; woman, Pharmacovigilance, prevalence.

\section{Introduction}

While medications usually provide considerable benefit in the maintenance of health, but medications may have a risk of adverse or unwanted effects, and evaluating the risk/balance benefit of a particular medication is a critical step in the decision to use pharmacotherapy. Adverse drug reactions (ADRs) are frequently associated with extended length of hospitalization, less cost effective patient care, and significant morbidity and mortality. ${ }^{1-3}$ Frequency of ADRs have increased with increase in number of medicines. ADRs were found responsible for $5 \%$ of all hospital admissions and found in 10-20\% of hospitalized patients. Early detection, assessment and monitoring of ADRs are must to decrease the morbidity to the patients and hence get better patient safety. ${ }^{4,5}$

The known risk factors for ADRs are increasing age, polypharmacy, associated renal and hepatic disease as well as being female. The incidence of ADRs is found to be higher than does the male gender. ${ }^{6}$ Female patients have a 1.5- to 1.7-fold greater risk of developing an ADR compared with male patients. It might be due to gender-related differences in pharmacokinetic, immunological and hormonal factors as well as differences in the use of medications by women in comparison to men. Women usually have a lower lean body mass, a reduced hepatic clearance, have differences in activity of cytochrome P450 enzymes and so metabolize drugs at different rates. ${ }^{7}$

So, due to increased evidences of ADRs in female, it becomes necessary that clinician should also evaluates the physiological and other cofactors to understand the high rate of adverse drug reaction in females.

Therefore, a retrospective analysis was planned out in this study to detect and assess the extent, pattern, severity and casualty by the ADRs in female patients of a tertiary hospital of Pauri Garhwal.

\section{Materials and Methods}

A retrospective study was conducted in the Department of Pharmacology of Veer Chandra Singh Garhwali Government Medical Science \& Research Institute and HNB Base \& Teaching Hospital, Srikot, Uttarakhand, India. The study was conducted after having approval from Institutional ethics committee, with the all suspected cases of ADRs in female patients of the various departments, of HNB Base \& Teaching Hospital, from $1^{\text {st } J a n u a r y ~} 2012$ to $31^{\text {st }}$ December 2015. Retrospective analysis was done for different concerned variables. We had excluded such female patients who developed ADRs following intake of any homeopathic, ayurvedic and indigenous medicine or in which we could not be identified suspected drug. Rechallenge was not done to any patient.

All the details like patient profile, type of reaction, suspect and concomitant drugs were documented in a prescribed format of Indian Pharmacopoeia Commission, Ghaziabad under the Pharmacovigilance Program of India ( Recent Suspected adverse drug reaction reporting form) ${ }^{8}$

Causality assessment was performed to find out the chances of relationship between a medicine and reaction which was mainly completed with the help of a 
questionnaire, Naranjo causality assessment algorithm which has categories like definite, probable, possible or doubtful relationship, on the basis of scoring. ${ }^{9}$

Data analysis was done by using appropriate methods and results were evaluated.

\section{Results}

In the various departments of HNB Base \& Teaching Hospital, total 206 patients suffered from ADRs during period of four years i.e. from 1st Jan 2012 to $31^{\text {st }}$ Dec 2015 . Out of which $110(53.4 \%)$ were female patients. Maximum number of ADRs were encountered in Dermatology followed by Medicine, Surgery, Obstetrics and Gynaecology, Psychiatry and Paediatrics departments.

On evaluating the data, the mean age of female patients affected due to ADRs was 31.53 years and the age group of $20-39$ years $(46,41.8 \%)$ were maximally affected ,followed by $40-59$ years $(29,26.4 \%)$. and 0-19 years $(24,21.8 \%)$. Most of affected patients (80, $72.72 \%)$ recovered within seven days whereas 18 (16.4\%) patients required 8 to 15 days to recover. We did not require any intervention in Seventy nine $(71.81 \%)$ cases but in $10(9.1 \%)$ cases some intervention were used for recovery.(Table 1) Drug used by oral routes were more frequently responsible for ADRs (38.18\%),followed by intravenous (33.63\%) routes.(Fig. 1) Department of Dermatology reported maximum ADRs(51, 46.36\%), followed by Medicine (26, 23.6\%) and Surgery $(19,17.02 \%)$ (Fig. 2). Amongst AMAs, beta lactams $(31,36.5 \%)$ quinolones $(17,20 \%)$ and aminoglycosides $(11,12.9 \%)$. were the most frequent causative medicines.

Skin as maximally involved $(76,69.1 \%)$ by ADRs in female patients, followed by gastrointestinal (GIT) $(16,14.5 \%)$, central nervous system (CNS) $(10,9.09 \%)$ and cardiovascular system (CVS) (5, 4.54\%). (Fig. 3). On evaluation of cutaneous manifestations, maximum number females were affected by exanthematous eruptions (EE), urticarial eruptions and pruritus.

The most common antibiotic resulting in ADRs was injection ceftriaxone $(20,18.18 \%)$, followed by tab ciprofloxacin $(7,6.3 \%)$. Causality assessment by Naranjo's scale illustrated that most of ADRs had probable $(58,52.7 \%)$ relationship and seventy six (52, $47.3 \%$ ) had possible relationship with the drugs.

Table 1: Descriptive table $(\mathbf{n}=110)$

\begin{tabular}{|l|c|c|}
\hline \multicolumn{2}{|l|}{ 1. Age distribution } \\
\hline Number (n) & Mean age & Std deviation \\
\hline 110 & 31.535 & 16.289 \\
\hline \multicolumn{2}{|l|}{ 2. Age range of patients } \\
\hline Age range & Frequency & Percentage \\
\hline $0-19$ & 24 & $21.8 \%$ \\
\hline $20-39$ & 46 & $41.8 \%$ \\
\hline $40-59$ & 29 & $26.4 \%$ \\
\hline $60-79$ & 11 & $10 \%$ \\
\hline
\end{tabular}

\begin{tabular}{|c|c|c|}
\hline Total & 110 & $100.0 \%$ \\
\hline \multicolumn{3}{|c|}{ 3. Male female ratios } \\
\hline Gender & Frequency & Percentage \\
\hline Female & 110 & $53.4 \%$ \\
\hline Male & 96 & $46.6 \%$ \\
\hline Total & 206 & $100.0 \%$ \\
\hline \multicolumn{3}{|c|}{ 4. Number of affected days } \\
\hline Duration & Frequency & Percentage \\
\hline $1-7$ days & 80 & $72.72 \%$ \\
\hline $8-15$ days & 18 & $16.4 \%$ \\
\hline $\begin{array}{l}\text { more than } 15 \\
\text { days }\end{array}$ & 12 & $10.9 \%$ \\
\hline Total & 110 & $100.0 \%$ \\
\hline \multicolumn{3}{|c|}{ 6. Different outcomes after ADRs } \\
\hline $\begin{array}{l}\text { Types of } \\
\text { Outcome }\end{array}$ & Frequency & Percentage \\
\hline Recovering & 20 & $18.2 \%$ \\
\hline Recovered & 79 & $71.81 \%$ \\
\hline $\begin{array}{l}\text { Required } \\
\text { intervention }\end{array}$ & 10 & $9.1 \%$ \\
\hline Unknown & 1 & $0.9 \%$ \\
\hline Total & 110 & $100.0 \%$ \\
\hline
\end{tabular}

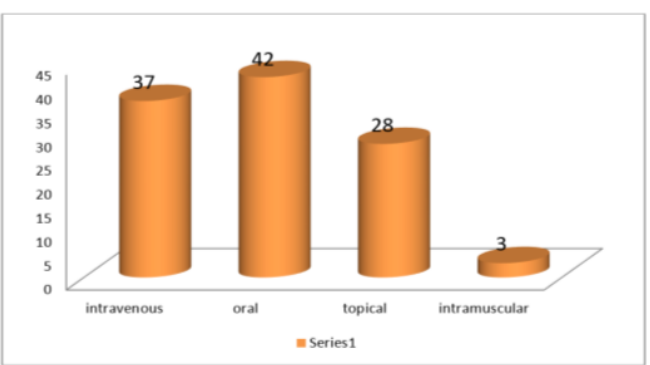

Fig. 1: Route of administration and ADRs

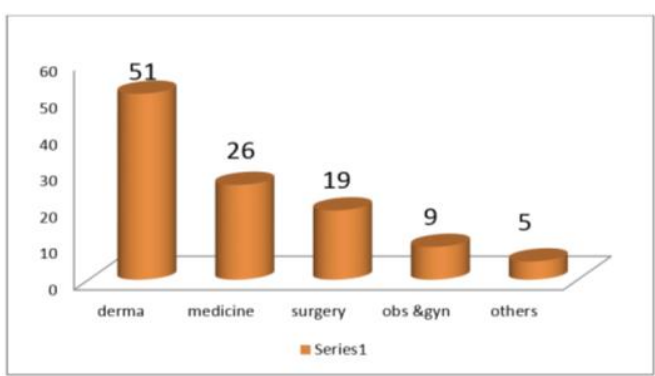

Fig. 2: Department wise distribution of Antimicrobials induced ADRs

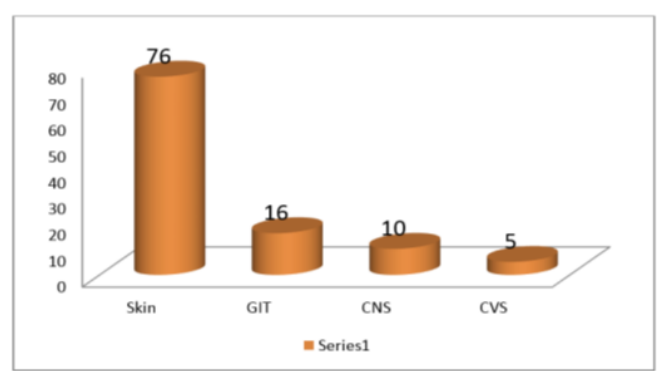

Figure 3: Systems involved in antimicrobial induced ADRs 


\section{Discussion}

Female gender experiences a higher chances of adverse drug reactions (ADRs) than does the male gender. A number of studies recommend that ADRs are 50 to $75 \%$ more likely in women than men. ${ }^{10-11}$ It has been suspected due to gender-related differences in pharmacokinetic, immunological and hormonal factors as well as differences in the use of medications by women in comparison to men.

In the present study, total 110 females were affected by ADR. Although this does not represent the true prevalence of ADR's because we had excluded the cases in which we could not find out the suspected medicine as well as those reactions which were due to herbal or homeopathic drugs. The Average age of the female patients in the study was 31.53 years, and female of age group of 20-39 affected maximally $(41.81 \%)$, which is in accordance to the result of previous studies. $^{12}$

It has been observed that less frequency of ADRs was there in younger girls than adult female like other study. ${ }^{13}$ It may be due to this explanation that usually lesser number of medicines are used in girls and girls of these age group usually have lesser hormonal interference in the physiological function of body which might affect the pharmacokinetic profile of medicines. Similarly Women use a considerably different range of medicines, particularly associated with oral contraception, menopause and pregnancy. ${ }^{14,15}$ As we know that oral contraceptives are mostly involved with drug interactions with a wide range of other therapeutic drugs. Even in other conditions like urinary tract infections, neurotic disorders, and arthritis, are also more prevalent in women. ${ }^{16}$ It is also well evident that women consume significantly more overthe-counter medications, herbal remedies and vitamins, compared with men. This increased rate of incidence might be due to more awareness of young female towards skin ailments and more reporting to physicians than the male. As in our part of the world, most of the medicines are easily available as over the counter drug therefore females who are usually very much enthuastic about self-medication, might be exposed to additional risk.

Most of the reactions were encountered for the duration between 1 to 21 days. Mostly patients (72.72\%) had reaction for seven days whereas $16.4 \%$ patients had symptoms for 8 to 15 days. So one week was sufficient for recovery of most of the symptoms. All the patients treated symptomatically for recovery from the symptoms. Ninety nine $(71.81 \%)$ cases did not require any intervention for recovery which represented that most of the ADRs were self limiting. Only dechallenge of the suspected medicine was enough to alleviate the reaction. So timely diagnosis of ADRs is of utmost importance for the improvement of patient safety.
Among the antimicrobials, maximum number of ADRs were mainly due to cephalosporins (36.5\%) whereas fluroquinolones were accountable in 17 cases $(20 \%)$. This increased rate of cephalosporins induced ADR's might be relating the irrational and more extensive use of these drugs as first-line or empirical therapy in this region. As antimicrobial resistance is another important issue due to irrational use of these medicines which is quiet alarming all over the world. All the physicians should be more vigilant for rational use of AMAs.

Similar results have been obtained in other studies where same group of antimicrobials like cephalosporins (34.69\%) fluoroquinolones (30.61\%), penicillins (14.28\%), were responsible for ADRs. ${ }^{17}$ The cephalosporins and fluoroquinolones were the most used antimicrobial in our set up, so it might be reason of more reported ADRs of these drug classes which is as accordance to another study. ${ }^{18}$

Similar type of results were observed in other studies where most common organs system involved were dermatological, gastrointestinal and central nervous system. ${ }^{19}$ Its very much evident that idiosyncratic drug reactions, particularly cutaneous reactions, appear to have an immunological etiology. It might be expected that gender difference in $\mathrm{T}$ cell activation and proliferation could be responsible for this as well as the increased prevalence of other skin diseases. $^{7}$

Causality assessment by Naranjo's scale revealed probable relationship in $58(52.71 \%)$ female patients and possible in about $52(47.3 \%)$ female patients with the suspected medicines which is similar to other studies. ${ }^{20}$ This evaluation of relationship between reactions and drugs could not be possible in polypharmacy cases.

The limitation of our study was less number of ADRs as we need a large scale study to evaluate the actual difference in the occurrence of ADRs between male and female As due to busy schedule of physicians in government hospitals it is not practically possible to report all the ADRs so it might be one of the reason of less number of ADRs in our study than actual prevalence rate.

\section{Conclusion}

Females definitely have a considerably more risk of developing adverse drug reactions than male counterparts. This seems to be due to number of physiological differences in women body along with dissimilarity in the medicine using behavior between men and women. So all the prescriber should be careful that gender difference might be a considerable factor in the prevalence of ADRs.

\section{References}

1. Suh DC, Woodall BS, Shin SK, Hermes-De Santis ER, Clinical and economic impact of adverse drug reactions 
in hospitalized patients, Ann Pharmacother,

2000;34:1373-79.

2. Johnson JA, Bootman JL., Drug-related morbidity and mortality. A cost-of-illness model, Arch Intern Med. 1995; 155:1949-56.

3. Classen DC, Pestotnik SL, Evans RS, Lloyd JF, Burke JP, Adverse drug events in hospitalized patients. Excess length of stay, extra costs, and attributable mortality, JAMA, 1997;277:301-6.

4. Pirmohamed M, Breckenridge AM, Kitteringham NR, Park BK, Adverse drug reactions, BMJ. 1998; 316: 1295 8.

5. Bates DW, Spell N, Cullen DJ, Burdick E, Laird N, Petersen LA, et al. The costs of adverse drug events in hospitalized patients. Adverse Drug Events Prevention Study Group. JAMA. 1997;277:307-11.

6. Zopf Y, Rabe C, Neubert A, Gassmann KG, Rascher W, Hahn EG, et al. Women encounter ADRs more often than do men. Eur J Clin Pharmacol. 2008;64(10):999-1004.

7. Rademaker M, Do women have more adverse drug reactions? Am J Clin Dermatol. 2001;2(6):349-51.

8. Available from: http:// www.ipc.gov.in/PvPI/pv_adr.html [last accessed on 2018 Jan 25]

9. Srinivasan R, Ramya G. Adverse drug reaction-causality assessment.IJRPC. 2011;1(3):606-12.

10. Pouyanne P, Haramburu F, Imbs JL, et al. Admissions to hospital caused by adverse drug reactions: cross sectional incidence study. BMJ 2000;320:1036.

11. Fattinger K, Roos M, Vergeres P, et al. Epidemiology of drug exposure and adverse drug reactions in two Swiss departments of internal medicine. Br J Clin Phar-macol 2000;49:158-67.

12. Pudukadan D,Thappa DM. Adverse cutaneous drug reactions: clinical pattern and causative agents in a tertiary care centre in south India.Indian J Dermatol Venereol Leprol. 2004; 70(1): 20-24.

13. Gonzalez Martin G, Caroca CM, Paris E. Adverse drug reactions (ADRs) in hospitalized pediatric patients. A prospective study. Int J Clin Pharmacol Ther. 1998;36(10):530-33.

14. Borda I, Jick H, Slone D, et al. Studies of drug usage in five Boston hospitals. JAMA 1967; 202: 506-10

15. Svarstad BL, Cleary PD, Mechanic D, et al. Gender differences in the acquisition of prescribed drugs: an epidemiological study. Med Care 1987;25:1089-98.

16. Sihvo S, Klaukka T, Martikainen J, et al. Frequency of daily over-the-counter drug use and potential clinically significant over-the-counter-prescription drug interactions in the Finnish adult population. Eur J Clin Pharmacol 2000;56:495-99.

17. Shamna M, Dilip C, Ajmal ,M , Mohan PL, Shinu C, Jafer CP et al. A prospective study on Adverse Drug Reactions of antibiotics in a tertiary care hospital. Saudi Pharmaceutical Journal 2014;22(4):303-8.

18. Stavreva G, Pendicheva D, Pandurska A, Marev R.Detection of adverse drug reactions to antimicrobial drugs in hospitalized patients Trakia J. Sci., 2008,6(1)79.

19. Horen B, Montastruc JL, Maryse. Mestre L. Adverse drug reaction \& off-label drug use in paediatric outpatients. $\mathrm{Br}$. J. Clin. Pharmacol.2002;54:665-70.

20. Priyadharsini R, Surendiran A, Adithan C, SreenivasanS, Sahoo FK. A study on adverse drug reactions in paediatric patients. J. Pharmacol. Pharmacother, 2011;2(4):277-80. 